\title{
ABSOLUTE CALIBRATION OF STELLAR TEMPERATURES
}

\author{
H. KIENLE \\ Ege University Observatory Izmir, Turkey \\ and \\ D. LABS \\ Landessternwarte Heidelberg-Königstuhl, F.R.G.
}

\begin{abstract}
The scale of effective temperatures $T_{\text {eff }}$ is based on observed absolute radiation temperatures $T_{r}$, which are defined by Planck's radiation law$$
i^{*}(\lambda) / i_{\mathrm{Au}}(\lambda)=\left[\exp \left(c_{2} / \lambda T_{\mathrm{Au}}\right)-1\right] /\left[\exp \left(c_{2} / \lambda T^{*}\right)-1\right],
$$

where $T_{\mathrm{Au}}$ designs the absolute temperature of the gold point. A relative scale of radiation temperatures can be derived from spectrophotometric comparisons with a standard star. The absolute calibration of the standard star ( $\alpha$ Lyr or Sun) demands a careful comparison with a standard radiation source of well known spectral energy distribution (Black Body or Synchrotron). With ground-based observations atmospheric extinction is to be taken into account; with extraterrestrial observations detectors may be used which are absolutely calibrated in a radiation laboratory under space conditions.
\end{abstract}

One of the relevant parameters which determine the physical state of a star is its effective temperature $T_{\text {eff }}$. It measures the total output of energy. With the Sun $T_{\text {eff }}$ can be derived from measures of the solar constant, with stars from absolute bolometric magnitudes. From spectrophotometric observations of stellar spectra we can only derive radiation temperatures $T_{r}$ based on the energy distribution of continuous spectra ('distribution temperature', 'gradient temperature'). The transition from observed $T_{r}$ to $T_{\text {eff }}$ is object of a theory of stellar atmospheres (model atmospheres).

The primary aim of observation must be the establishing of a scale of absolute radiation temperatures based on the fundamental definition

$$
\frac{i_{*}(\hat{\lambda})}{i_{\mathrm{Au}}(\lambda)}=\frac{\exp \left(c_{2} / \lambda T_{\mathrm{Au}}\right)-1}{\exp \left(c_{2} / \lambda T_{*}\right)-1},
$$

where $T_{\mathrm{Au}}$ denotes the temperature of melting gold on the thermodynamic scale ('gold point')

The problem of calibration. We have to compare the radiation of a star with a primary standard of radiation of known absolute temperature. The block scheme of Figure 1 shows how such a comparison may be performed. The radiation of star $S$ after passing interstellar space meets a detector D (telescope + photometric device) either at a ground-based or an extraterrestrial orbiting observatory. If the absolute response curve of the whole detector arrangement is known from comparison with primary standard radiation we get from spectrophotometric observations the absolute intensity

$$
J(\lambda) \Delta \lambda=\frac{1}{r^{2}} \int_{\Delta \lambda} J^{*}(\lambda) p_{i}(\lambda) p_{a}(\lambda) \mathrm{d} \lambda,
$$

B. Hauck and B.E. Westerlund (eds.), Problems of Calibration of Absolute Magnitudes and Temperature of Stars, 153-155. All Rights Reserved. Copyright (C) 1973 by the IAU. 
where $r=$ distance, $p_{i}(\lambda)$ interstellar absorption, and $p_{a}(\lambda)$ atmospheric extinction. If the transmission coefficient of the terrestrial atmosphere is carefully determined (for the time of observation!) only interstellar absorption ('reddening') remains as a possible source of systematic error of observed temperatures $T(\lambda)$.

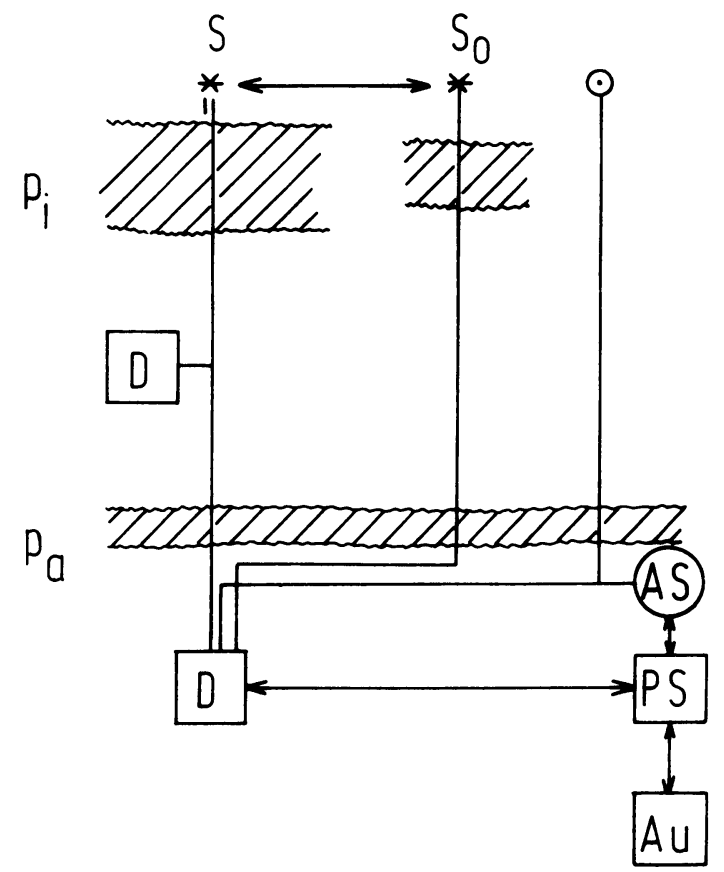

Fig. 1. $\mathbf{S}=$ star, $\mathrm{S}_{0}=$ standard star, $\odot=$ sunlike star, $p_{i}=$ interstellar absorption, $p_{a}=$ atmospheric absorption, $\mathrm{D}=$ detector, $\mathbf{A S}=$ artificial star, $\mathbf{P S}=$ primary standard, $\mathrm{Au}=$ gold point .

Establishing the temperature scale can be split up into two parts:

(a) By intercomparison of stars of different spectral types with a standard star $\mathrm{S}_{0}$ we get a scale of relative temperatures $T / T_{0}$.

(b) Absolute calibration of the standard star allows the transition from $T / T_{0}$ to $a b$ solute temperature $T$.

As standard stars spectral type A0 stars (especially $\alpha$ Lyr) have been used in the past. It would perhaps be preferable to choose early B stars ( $\eta \mathrm{U} \mathrm{Ma}, \mathrm{S}$ Mon) because of the better definition of the continuum, correction for lines being smaller.

The absolute temperature scale derived from comparison with a standard star can be checked by data from absolute calibration of the Sun which is to be regarded as a representative G2V star with known spectral energy distribution.

Primary standard radiation sources are the Black Body (BB) at about $3000 \mathrm{~K}$ for wavelengths larger than $2000 \AA$ and the Synchrotron for the extreme UV below $3600 \AA$. Both must be calibrated by comparison with BB-radiation at fix points of the thermodynamic temperature scale (melting point of gold or copper). The overlapping part of 
the energy curves between $2000 \AA$ and $3600 \AA$ offer a useful checking of the absolute values.

Absolute calibration may be performed either by deriving the absolute response curve of the whole detector arrangement or by interference of an 'artificial star' of well known spectral energy distribution. Often the response curve has been calculated from its different components (absorption by optical elements, sensitivity of photometric elements). No doubt it will be better to calibrate the whole detector in the laboratory with help of primary or secondary standards.

If a well calibrated artificial star is used for comparison, the whole detector arrangement will be eliminated ('zero method').

Calibration Work at Heidelberg Radiation Laboratory

(a) Absolute spectral energy of Sun's radiation from $\lambda=0.3 \mu$ to $1.2 \mu$. Standard value of solar constant.

(b) Blackbody radiation for $T=3000 \mathrm{~K}$.

(c) Comparison Synchrotronradiation - Blackbody.

(d) Secondary standards for the region from 0.16 to $10 \mu$.

(e) Artificial star. 